\title{
GENERALIZED STATISTICAL CONVERGENCE OF DOUBLE SEQUENCES IN PARANORMED SPACES
}

\section{ABDUllah AlOtAiBI ${ }^{1,2}$, AlAA MOHAMMED ALJAHILI ${ }^{2}$ AND S. A. MOHIUDDINE ${ }^{1, *}$}

${ }^{1}$ Operator Theory and Applications Research Group, Department of Mathematics, Faculty of Science, King Abdulaziz University, P.O. Box 80203, Jeddah 21589, Saudi Arabia

${ }^{2}$ Department of Mathematics, Faculty of Science, King Abdulaziz University, Jeddah, Saudi Arabia

*Corresponding author: mohiuddine@gmail.com

\begin{abstract}
We introduce the notion of $(\lambda, \mu)$-statistical convergence of double sequences in a setting of paranormed space and prove that every convergent sequence is $(\lambda, \mu)$-statistically convergent but not conversely by supporting an illustrative example. We also define the notions of $(\lambda, \mu)$-statistical Cauchy and strongly $(\lambda, \mu)_{p}$-summable double sequences in a paranormed space and obtain their relationship with $(\lambda, \mu)$ statistical convergence.
\end{abstract}

\section{INTRODUCTION AND PRELIMINARIES}

The notion of statistical convergence, which is an extension of the idea of common convergence, was first appeared, under the name of almost convergence, in the first edition of the celebrated monograph of Zygmund [32]. This idea was introduced by Fast [11] as follows: The sequence $x=\left(x_{k}\right)$ is statistically convergent to $\ell$ if for every $\varepsilon>0, \lim _{n} n^{-1}\left|\left\{k \leq n:\left|x_{k}-\ell\right| \geq \varepsilon\right\}\right|=0$. Some basic properties of statistical convergence were studied by Schoenberg [30], Šalát [31] and Connor [9]. An interesting notion of statistically

Received 2019-01-17; accepted 2019-02-21; published 2019-09-02.

2010 Mathematics Subject Classification. 40A05, 40G05.

Key words and phrases. double sequence; statistical convergence; statistical Cauchy; strong p-Cesàro summability; paranormed space.

(C)2019 Authors retain the copyrights of their papers, and all open access articles are distributed under the terms of the Creative Commons Attribution License. 
Cauchy sequence was first defined by Fridy [12] and he also showed that it is equivalent to statistical convergence. Thereafter, this notion turned out to be one of the most active areas of research in summability theory. The statistical convergence was studied in various setup such as topological Hausdorff groups [8], normed spaces [15], locally convex Hausdorff topological spaces [16], paranormed spaces [2], random 2-normed spaces [19] and many others. Mursaleen [24] presented a generalization of statistical convergence with the help of non-decreasing sequence $\lambda=\left(\lambda_{k}\right)$ such that $\lambda_{k+1} \leq \lambda_{k}+1$ and $\lambda_{1}=0$ of positive numbers tending to $\infty$ and called it $\lambda$-statistical convergence. We also refer to the recent work in $[1,3,4,6,7,10,14,17,18,21,22]$ for some applications of convergence methods to approximation theorems. Pringsheim [29] extended the notion of usual convergence from single sequences of real numbers to double sequences as follows: A double sequence $x=\left(x_{j k}\right)$ has a Pringsheim limit $\xi$ (convergent to $\xi$ in Pringsheim's sense), in symbols, we shall write $(P) \lim x=\xi$, provided that given an $\epsilon>0$ there exists an $N \in \mathbb{N}$ such that $\left|x_{j k}-\xi\right|<\epsilon$ whenever $j, k>N$. Also, $x=\left(x_{j k}\right)$ is bounded, denoted by $\mathcal{L}_{\infty}$, if

$$
\|x\|=\sup _{j, k}\left|x_{j k}\right|<\infty
$$

It is well known that every convergent single sequence is bounded but this fact need not be true for double sequences. Statistical convergence extended to double sequences by Mursaleen and Edely [26] with the help of two dimensional analogue of natural density of subsets of $\mathbb{N} \times \mathbb{N}$ and further it was defined and studied in intuitionistic fuzzy normed spaces, locally solid Riesz spaces and paranormed spaces by Mursaleen and Mohiuddine [27,28], Mohiuddine et al. [20] and Arani et al. [5], respectively. Also, we refer to [13,23]. Let $K \subset \mathbb{N} \times \mathbb{N}$. Then, the double natural density of $K$ is defined by

$$
\delta_{2}(K)=(P) \lim _{m, n} \frac{|K(m, n)|}{m n}
$$

provided that the limit exists, where $|K(m, n)|$ be the numbers of $(j, k)$ in $K$ such that $j \leq m$ and $k \leq n$. $x=\left(x_{j k}\right)$ is said to be statistically convergent to $\xi$ if for each $\epsilon>0$,

$$
(P) \lim _{m, n}(m n)^{-1}\left|\left\{(j, k), j \leq m, k \leq n:\left|x_{j k}-\xi\right| \geq \epsilon\right\}\right|=0
$$

Mursaleen et al. [25] defined and studied the notion of $(\lambda, \mu)$-statistical convergence for double sequences where $\lambda=\lambda_{m}$ and $\mu=\mu_{n}$ are two non-decreasing sequences of positive real numbers each tending to $\infty$ such that $\lambda_{1}=0, \lambda_{m+1} \leq \lambda_{m}+1$ and $\mu_{1}=0, \mu_{n+1} \leq \mu_{n}+1$ for all $m, n$. The $(\lambda, \mu)$-density of the set $K \subseteq \mathbb{N} \times \mathbb{N}$ is given by

$$
\delta_{\lambda, \mu}(K)=(P) \lim _{m, n} \frac{1}{\lambda_{m} \mu_{n}}\left|\left\{m-\lambda_{m}+1 \leq j \leq m, n-\mu_{n}+1 \leq k \leq n:(j, k) \in K\right\}\right|
$$


provided that the limit exists. We remark that $\lambda_{m}=m$ and $\mu_{n}=n$, the $(\lambda, \mu)$-density reduces to the natural double density. A double sequence $x=\left(x_{j k}\right)$ is $(\lambda, \mu)$-statistically convergent to $\xi$ if for every $\epsilon>0$,

$$
\text { (P) } \lim _{m, n} \frac{1}{\lambda_{m} \mu_{n}}\left|\left\{(j, k), j \in I_{m}, k \in J_{n}:\left|x_{j k}-\xi\right| \geq \epsilon\right\}\right|=0
$$

where $I_{m}=\left[m-\lambda_{m}+1, m\right]$ and $J_{n}=\left[n-\mu_{n}+1, n\right]$.

If $X$ is a linear space and $g: X \rightarrow \mathbb{R}$ such that (i) $x=0 \Rightarrow g(x)=0$, (ii) $g(x+y) \leq g(x)+g(y)$, (iii) $g(-x)=g(x)$ and (iv) if $t_{k} \rightarrow t(k \rightarrow \infty)$ and $x_{k} \rightarrow x(k \rightarrow \infty)$ in the sense that $g\left(x_{k}-x\right) \rightarrow 0(k \rightarrow \infty)$ for scalars $t_{k}, t$ and the vectors $x_{k}, x \in X$, then $t_{k} x_{k} \rightarrow t x(k \rightarrow \infty)$ in the sense that $g\left(t_{k} x_{k}-t x\right) \rightarrow 0$ $(k \rightarrow \infty)$, then $g$ is said to be a paranorm on $X$ and the pair $(X, g)$ is called a paranormed space. Note that if $g(x)=0$ implies $x=0$, then paranorm $g$ is called a total paranorm on $X$ and the pair $(X, g)$ is called a total paranormed space. It is to further note that each seminorm (norm) on $X$ is a paranorm (total) but not conversely.

\section{2. $(\lambda, \mu)$-Statistical CONVERGENCE IN PARANORMED SPACES}

In this section, we introduce the notion of convergence and $(\lambda, \mu)$-statistical convergence in the framework of paranormed space and prove various interesting results and display an illustrative example is support of our result.

Definition 2.1. Let $(X, g)$ be a paranormed space. We say that a double sequence $x=\left(x_{j k}\right)$ is convergent, shortly, $g_{2}$-convergent, to $\xi$ in $(X, g)$ if for each $\epsilon>0$, there is $k_{0} \in \mathbb{N}$ such that $g\left(x_{j k}-\xi\right)<\epsilon$ whenever $j, k \geq k_{0}$. In symbols, one writes $g_{2}-\lim x=\xi$, and $\xi$ is called the $g_{2}$-limit of $x$.

Definition 2.2. Let $(X, g)$ be a paranormed space. We say that a double sequence $x=\left(x_{j k}\right)$ is $(\lambda, \mu)$ statistically convergent, shortly, $g\left(S_{\lambda, \mu}\right)$-convergent, to $\xi$ in $(X, g)$, if for each $\epsilon>0$, the set $\{(j, k) \in \mathbb{N} \times \mathbb{N}$ : $\left.g\left(x_{j k}-\xi\right) \geq \epsilon\right\}$ has $(\lambda, \mu)$-density zero, equivalently, one writes

$$
(P) \lim _{m, n} \frac{1}{\lambda_{m} \mu_{n}}\left|\left\{(j, k), j \in I_{m}, k \in J_{n}: g\left(x_{j k}-\xi\right) \geq \epsilon\right\}\right|=0 .
$$

In this case, one writes $g\left(S_{\lambda, \mu}\right)-\lim x=\xi$. If we choose $\lambda_{m}=m$ and $\mu_{n}=n$ then the notion of $g\left(S_{\lambda, \mu}\right)$ convergence is reduced to statistically convergence for double sequence in $(X, g)$ due to Arani et al. [5]. We denote this by $g\left(S_{2}\right)$-convergence and write $g\left(S_{2}\right)-\lim x=\xi$.

Theorem 2.3. If a double sequence $x=\left(x_{j k}\right)$ is $g\left(S_{\lambda, \mu}\right)$-convergent then $g\left(S_{\lambda, \mu}\right)$-limit is unique.

Proof. Assume that $g\left(S_{\lambda, \mu}\right)-\lim x=\xi^{\prime}$ and $g\left(S_{\lambda, \mu}\right)-\lim x=\xi^{\prime \prime}$. Let $\epsilon>0$ be given. We now define the following two sets $B^{\prime}(\epsilon)=\left\{(j, k) \in \mathbb{N} \times \mathbb{N}: g\left(x_{j k}-\xi^{\prime}\right) \geq \epsilon / 2\right\}$ and $B^{\prime \prime}(\epsilon)=\left\{(j, k) \in \mathbb{N} \times \mathbb{N}: g\left(x_{j k}-\xi^{\prime \prime}\right) \geq \epsilon / 2\right\}$. 
Since $g\left(S_{\lambda, \mu}\right)-\lim x=\xi^{\prime}$, one obtains

$$
\delta_{\lambda, \mu}\left(B^{\prime}(\epsilon)\right)=(P) \lim _{m, n} \frac{1}{\lambda_{m} \mu_{n}}\left|\left\{(j, k), j \in I_{m}, k \in J_{n}: g\left(x_{j k}-\xi^{\prime}\right) \geq \epsilon / 2\right\}\right|=0 .
$$

Similarly, the assumption $g\left(S_{\lambda, \mu}\right)-\lim x=\xi^{\prime \prime}$ gives $\delta_{\lambda, \mu}\left(B^{\prime \prime}(\epsilon)\right)=0$. Now, let $B(\epsilon)=B^{\prime}(\epsilon) \cup B^{\prime \prime}(\epsilon)$. Then $\delta_{\lambda, \mu}(B(\epsilon))=0$ and so $\delta_{\lambda, \mu}\left(B^{c}(\epsilon)\right)=1$ since $B^{c}(\epsilon)$ is a nonempty set. Now if $(j, k) \in \mathbb{N} \times \mathbb{N} \backslash B(\epsilon)$, then

$$
g\left(\xi^{\prime}-\xi^{\prime \prime}\right) \leq g\left(x_{j k}-\xi^{\prime}\right)+g\left(x_{j k}-\xi^{\prime \prime}\right)<\epsilon / 2+\epsilon / 2=\epsilon .
$$

Since $\epsilon>0$ is arbitrary, one obtains $g\left(\xi^{\prime}-\xi^{\prime \prime}\right)=0$ which yields $\xi^{\prime}=\xi^{\prime \prime}$.

Theorem 2.4. If a double sequence $x=\left(x_{j k}\right)$ is $g_{2}$-convergent to $\xi$, then it is $g\left(S_{\lambda, \mu}\right)$-convergent to the same limit.

Proof. Assume that $\left(x_{j k}\right)$ is $g_{2}$-convergent to $\xi$, that is, $g_{2}$ - $\lim x=\xi$. Let $\epsilon>0$ be given. Then, there is $N \in \mathbb{N}$ such that $g\left(x_{j k}-\xi\right)<\epsilon$ for all $j, k \geq N$. Since, the set $K(\epsilon)=\left\{(j, k) \in \mathbb{N} \times \mathbb{N}: g\left(x_{j k}-\xi\right) \geq \epsilon\right\}$ is contained in $\mathbb{N} \times \mathbb{N}$, hence $\delta_{\lambda, \mu}(K(\epsilon))=0$, that is, $\left(x_{j k}\right)$ is $g\left(S_{\lambda, \mu}\right)$-convergent to $\xi$.

Example 2.5. The present example proves that the converse of last Theorem is not true in general. Let

$$
X=\ell(1 / j k)=\left\{x=\left(x_{j k}\right): \sum_{j=1}^{\infty} \sum_{k=1}^{\infty}\left|x_{j k}\right|^{1 / j k}<\infty\right\}
$$

with the paranorm

$$
g(x)=\sum_{j=1}^{\infty} \sum_{k=1}^{\infty}\left|x_{j k}\right|^{1 / j k}
$$

Let us define $x=\left(x_{j k}\right)$ by

$$
x_{j k}=\left\{\begin{array}{l}
j k \text { for } m-\left[\sqrt{\lambda_{m}}\right]+1 \leq j \leq m, n-\left[\sqrt{\mu_{n}}\right]+1 \leq k \leq n, \\
0 \text { otherwise. }
\end{array}\right.
$$

For $0<\epsilon<1$, one writes

$$
B(\epsilon):=\left\{(j, k) \in \mathbb{N} \times \mathbb{N}: g\left(x_{j k}\right) \geq \epsilon\right\} .
$$

It is easy to see that

$$
g\left(x_{j k}\right)=\left\{\begin{array}{l}
(j k)^{1 / j k} \text { for } m-\left[\sqrt{\lambda_{m}}\right]+1 \leq j \leq m, n-\left[\sqrt{\mu_{n}}\right]+1 \leq k \leq n, \\
0 \text { otherwise }
\end{array}\right.
$$

and hence we obtain

$$
(P) \lim _{j k} g\left(x_{j k}\right):=\left\{\begin{array}{l}
1 \text { for } m-\left[\sqrt{\lambda_{m}}\right]+1 \leq j \leq m, n-\left[\sqrt{\mu_{n}}\right]+1 \leq k \leq n, \\
0 \text { otherwise. }
\end{array}\right.
$$


We obtain that $\left(x_{j k}\right)$ is not convergent in $(X, g)\left(g_{2}\right.$ - $\lim x$ does not exist) but $\delta_{\lambda, \mu}(B(\epsilon))=0$, that is, $g\left(S_{\lambda, \mu}\right)$ - $\lim x=0$. Hereby, we conclude that the converse of above Theorem 2.4 need not be true in general.

The proof of the following theorem is straightforward and hence omitted.

Theorem 2.6. Let $(X, g)$ be a paranormed space and assume that $g\left(S_{\lambda, \mu}\right)-\lim x^{\prime}=\xi^{\prime}$ and $g\left(S_{\lambda, \mu}\right)-\lim x^{\prime \prime}=$ $\xi^{\prime \prime}$. Then

(i) $g\left(S_{\lambda, \mu}\right)-\lim \left(x^{\prime} \pm x^{\prime \prime}\right)=\xi^{\prime} \pm \xi^{\prime \prime}$,

(ii) $g\left(S_{\lambda, \mu}\right)-\lim \alpha x^{\prime}=c \xi^{\prime}$ for $c \in \mathbb{R}$.

Theorem 2.7. Let $(X, g)$ be a paranormed space. Then, a double sequence $x=\left(x_{j k}\right)$ is $(\lambda, \mu)$-statistically convergent to $\xi$ in $(X, g)$ if and only if there exists a set $B=\left\{\left(j_{m}, k_{n}\right): j_{1}<j_{2}<\ldots<j_{m}<\ldots ; k_{1}<k_{2}<\right.$ $\left.\ldots<k_{n}<\ldots\right\} \subseteq \mathbb{N} \times \mathbb{N}$ with $\delta(B)=1$ such that $g-\lim _{m, n} x_{j_{m} k_{n}}=\xi$.

Proof. Suppose that $x=\left(x_{j k}\right)$ is $g\left(S_{\lambda, \mu}\right)$-convergent to $\xi$, that is, $g\left(S_{\lambda, \mu}\right)$ - $\lim x=\xi$. For $s=1,2, \ldots$, one writes

$$
B(s)=\left\{(m, n) \in \mathbb{N} \times \mathbb{N}: g\left(x_{j_{m} k_{n}}-\xi\right) \geq \frac{1}{s}\right\}
$$

and

$$
D(s)=\left\{(m, n) \in \mathbb{N} \times \mathbb{N}: g\left(x_{j_{m} k_{n}}-\xi\right)<\frac{1}{s}\right\}
$$

Then $\delta_{\lambda, \mu}(B(s))=0$,

$$
D(1) \supset D(2) \supset \ldots \supset D(i) \supset D(i+1) \supset \ldots
$$

and

$$
\delta_{\lambda, \mu}(D(s))=1(s=1,2, \ldots) .
$$

We need to prove that $\left(x_{j_{m} k_{n}}\right)$ is $g_{2}$-convergent to $\xi$ for $(m, n) \in D(s)$. Let us assume, on contrary, that $\left(x_{j_{m} k_{n}}\right)$ is not $g_{2}$-convergent to $\xi$. Consequently, there is $\epsilon>0$ such that $g\left(x_{k_{n}}-\xi\right) \geq \epsilon$ for infinitely many terms. Let us write

$$
D(\epsilon)=\left\{(m, n) \in \mathbb{N} \times \mathbb{N}: g\left(x_{j_{m} k_{n}}-\xi\right)<\epsilon\right\}
$$

and $\epsilon>\frac{1}{s}, s \in \mathbb{N}$. Then

$$
\delta_{\lambda, \mu}(D(\epsilon))=0
$$

and by $(1), D(s) \subset D(\epsilon)$. Hence $\delta_{\lambda, \mu}(D(s))=0$, which contradicts $(2)$ and therefore $\left(x_{j_{m} k_{n}}\right)$ is $g_{2}$-convergent to $\xi$. 
Conversely, let us assume there exists a set $B=\left\{\left(j_{m}, k_{n}\right): j_{1}<j_{2}<\ldots<j_{m}<\ldots ; k_{1}<k_{2}<k_{3}<\right.$ $\left.\ldots<k_{n}<\ldots\right\} \subseteq \mathbb{N} \times \mathbb{N}$ with $\delta(B)=1$ such that $g-\lim _{n \rightarrow \infty} x_{j_{m} k_{n}}=\xi$. Then there is a positive integer $N$ such that $g\left(x_{m n}-\xi\right)<\epsilon$ for $m, n>N$. Put

$$
B(\epsilon)=\left\{(m, n) \in \mathbb{N} \times \mathbb{N}: g\left(x_{m n}-\xi\right) \geq \epsilon\right\}
$$

and $B^{\prime}=\left\{\left(j_{N+1}, k_{N+1}\right),\left(j_{N+2}, k_{N+2}\right), \ldots\right\}$. Then $\delta_{\lambda, \mu}\left(B^{\prime}\right)=1$ and $B(\epsilon) \subseteq \mathbb{N} \times \mathbb{N}-B^{\prime}$ which implies that $\delta_{\lambda, \mu}(B(\epsilon))=0$. Hence $g\left(S_{\lambda, \mu}\right)-\lim x=\xi$.

We are now defining the notion of $(\lambda, \mu)$-statistically Cauchy double sequence in a paranormed space and prove that it is equivalent to the notion of $(\lambda, \mu)$-statistically convergence double sequence.

Definition 2.8. Let $(X, g)$ be a paranormed space. We say that $x=\left(x_{j k}\right)$ is $(\lambda, \mu)$-statistically Cauchy double sequence in $(X, g)$, denoted by $g\left(S_{\lambda, \mu}\right)$-Cauchy, if for every $\epsilon>0$ there exist $M, N \in \mathbb{N}$ such that, for all $j, m \geq M, k, n \geq N$, we have

$$
(P) \lim _{m, n} \frac{1}{\lambda_{m} \mu_{n}}\left|\left\{(j, k), j \in I_{m}, k \in J_{n}: g\left(x_{j k}-x_{m n}\right) \geq \epsilon\right\}\right|=0 .
$$

Theorem 2.9. Let $x=\left(x_{j k}\right)$ be a double sequence in a complete paranormed space $(X, g)$. Then, $x$ is $g\left(S_{\lambda, \mu}\right)$-convergent iff it is $g\left(S_{\lambda, \mu}\right)$-Cauchy.

Proof. Assume that $g\left(S_{\lambda, \mu}\right)-\lim x=\xi$. Then, the set

$$
G(\epsilon)=\left\{(j, k) \in \mathbb{N} \times \mathbb{N}: g\left(x_{j k}-\xi\right) \geq \epsilon / 2\right\}
$$

has $(\lambda, \mu)$-density zero which yields

$$
\delta_{\lambda, \mu}\left(G^{c}(\epsilon)\right)=\delta_{\lambda, \mu}\left(\left\{(j, k) \in \mathbb{N} \times \mathbb{N}: g\left(x_{j k}-\xi\right)<\epsilon\right\}\right)=1 .
$$

Suppose $(m, n) \in G^{c}(\epsilon)$. Therefore, $g\left(x_{m n}-\xi\right)<\epsilon / 2$. Now, let

$$
H(\epsilon):=\left\{(j, k) \in \mathbb{N} \times \mathbb{N}: g\left(x_{j k}-x_{m n}\right) \geq \epsilon\right\}
$$

We need to show that $H(\epsilon) \subset G(\epsilon)$. Let $(j, k) \in H(\epsilon)$. Then $g\left(x_{j k}-x_{m n}\right) \geq \epsilon$ and hence $g\left(x_{j k}-\xi\right) \geq \epsilon / 2$, i.e., $(j, k) \in G(\epsilon)$. Otherwise, if $g\left(x_{j k}-\xi\right)<\varepsilon$ then

$$
\epsilon \leq g\left(x_{j k}-x_{m n}\right) \leq g\left(x_{j k}-\xi\right)+g\left(x_{m n}-\xi\right)<\frac{\epsilon}{2}+\frac{\epsilon}{2}=\epsilon,
$$

which is not possible. Thus $H(\epsilon) \subset G(\epsilon)$ and hence

$$
\delta_{\lambda, \mu}(H(\epsilon))=\delta_{\lambda, \mu}\left(\left\{(j, k) \in \mathbb{N} \times \mathbb{N}: g\left(x_{j k}-x_{m n}\right) \geq \epsilon\right\}\right)=0 .
$$

Therefore $\left(x_{j k}\right)$ is $(\lambda, \mu)$-statistically Cauchy in $(X, g)$. 
Conversely, assume that $x=\left(x_{j k}\right)$ is $g\left(S_{\lambda, \mu}\right)$-Cauchy but not $g\left(S_{\lambda, \mu}\right)$-convergent. Then there exist $M, N \in \mathbb{N}$ such that for all $j, m \geq M, k, n \geq N$, the set

$$
A(\epsilon)=\left\{(j, k) \in \mathbb{N} \times \mathbb{N}: g\left(x_{j k}-x_{m n}\right) \geq \epsilon\right\},
$$

has $(\lambda, \mu)$-density zero, that is, $\delta_{\lambda, \mu}\left(A(\epsilon)=0\right.$ and $\delta_{\lambda, \mu}(E(\epsilon))=0$, where

$$
E(\epsilon)=\left\{(j, k) \in \mathbb{N} \times \mathbb{N}: g\left(x_{j k}-\xi\right)<\frac{\epsilon}{2}\right\},
$$

that is,

$$
\text { (P) } \lim _{m, n} \frac{1}{\lambda_{m} \mu_{n}}\left|\left\{(j, k), j \in I_{m}, k \in J_{n}: g\left(x_{j k}-\xi\right)<\frac{\epsilon}{2}\right\}\right|=0,
$$

which yields $\delta_{\lambda, \mu}\left(E^{c}(\epsilon)\right)=1$. Since $g\left(x_{j k}-x_{m n}\right) \leq 2 g\left(x_{j k}-\xi\right)<\epsilon$, if $g\left(x_{j k}-\xi\right)<\epsilon / 2$. Thus, $\delta_{\lambda, \mu}\left(A^{C}(\epsilon)\right)=0$ and so $\delta_{\lambda, \mu}\left(A(\epsilon)=1\right.$. This is a contradiction to our assumption that $x$ is $g\left(S_{\lambda, \mu}\right)$-Cauchy. Hence $x$ is $g\left(S_{\lambda, \mu}\right)$ convergent.

\section{Strong summability for double Sequences in $(X, g)$}

We give the idea of strong $(\lambda, \mu)_{p}$-summability in the setting of paranormed space $(X, g)$ and obtain its relation with $g\left(S_{\lambda, \mu}\right)$-convergence.

Definition 3.1. Let $(X, g)$ be a paranormed space and let $p$ be a positive real number. The double sequence

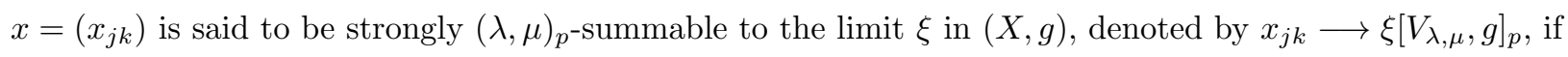

$$
(P) \lim _{m, n} \frac{1}{\lambda_{m} \mu_{n}} \sum_{j \in I_{m}} \sum_{k \in J_{n}}\left(g\left(x_{j k}-\xi\right)\right)^{p}=0(0<p<\infty) .
$$

Theorem 3.2. One has the following:

(i) If $0<p<\infty$ and $x_{j k} \longrightarrow \xi\left[V_{\lambda, \mu}, g\right]_{p}$, then $x=\left(x_{j k}\right)$ is $g\left(S_{\lambda, \mu}\right)$-convergent to $\xi$.

(ii) If $x=\left(x_{j k}\right) \in \mathcal{L}_{\infty}$ and $g\left(S_{\lambda, \mu}\right)$-convergent to $\xi$ then $x_{j k} \longrightarrow \xi\left[V_{\lambda, \mu}, g\right]_{p}$, where $p \in(0, \infty)$.

Proof. (i) Assume that $x_{j k} \longrightarrow \xi\left[V_{\lambda, \mu}, g\right]_{p}$. Then, as $m, n \rightarrow \infty$, one obtains

$$
\begin{gathered}
0 \longleftarrow \frac{1}{\lambda_{m} \mu_{n}} \sum_{j \in I_{m}} \sum_{k \in J_{n}}\left(g\left(x_{j k}-\xi\right)\right)^{p} \geq \frac{1}{\lambda_{m} \mu_{n}} \sum_{\substack{j \in I_{m} \\
\left(g\left(x_{k}-\xi\right)\right)^{p} \geq \epsilon}} \sum_{\substack{k \in J_{n} \\
\left(g\left(x_{k}-\xi\right)\right)^{p} \geq \epsilon}}\left(g\left(x_{k}-\xi\right)\right)^{p} \\
\geq \frac{\epsilon^{p}}{\lambda_{m} \mu_{n}}|F(\epsilon)|,
\end{gathered}
$$

where $F(\epsilon)=\left\{j \in I_{m}, k \in J_{n}:\left(g\left(x_{j k}-\xi\right)\right)^{p} \geq \epsilon\right\}$. That is, $(P) \lim _{m, n \rightarrow \infty} \frac{1}{\lambda_{m} \mu_{n}}|F(\epsilon)|=0$ and so

$$
\delta_{\lambda, \mu}(F(\epsilon))=(P) \lim _{m, n} \frac{1}{\lambda_{m} \mu_{n}}\left|\left\{(j, k), j \in I_{m}, k \in J_{n}:\left(g\left(x_{j k}-\xi\right)\right)^{p} \geq \epsilon\right\}\right|=0 .
$$

Thus $x=\left(x_{j k}\right)$ is $g\left(S_{\lambda, \mu}\right)$-convergent to $\xi$. 
(ii) Assume that a double sequence $x=\left(x_{j k}\right)$ is bounded and $g\left(S_{\lambda, \mu}\right)$-convergent to $\xi$. Let $\epsilon>0$ be given. Then, we have $\delta_{\lambda, \mu}(F(\epsilon))=0$. Since $x \in \mathcal{L}_{\infty}$, there is an $M>0$ such that $g\left(x_{j k}-\xi\right) \leq M$. We have

$$
\begin{gathered}
\frac{1}{\lambda_{m} \mu_{n}} \sum_{j \in I_{m}} \sum_{k \in J_{n}}\left(g\left(x_{j k}-\xi\right)\right)^{p} \\
=\frac{1}{\lambda_{m} \mu_{n}} \sum_{\substack{j \in I_{m} \\
(j, k) \notin F(\epsilon)}} \sum_{\substack{k \in J_{n} \\
(j, k) \notin F(\epsilon)}}\left(g\left(x_{j k}-\xi\right)\right)^{p}+\frac{1}{\lambda_{m} \mu_{n}} \sum_{\substack{j \in I_{m} \\
(j, k) \in F(\epsilon)}} \sum_{\substack{k \in J_{n} \\
(j, k) \in F(\epsilon)}}\left(g\left(x_{j k}-\xi\right)\right)^{p} .
\end{gathered}
$$

If we take $(j, k) \notin F(\epsilon)$ then

$$
\frac{1}{\lambda_{m} \mu_{n}} \sum_{\substack{j \in I_{m} \\(j, k) \notin F(\epsilon)}} \sum_{\substack{k \in J_{n} \\(j, k) \notin F(\epsilon)}}\left(g\left(x_{j k}-\xi\right)\right)^{p}<\epsilon .
$$

On the other and, if $(j, k) \in F(\epsilon)$, we have

$$
\begin{gathered}
\frac{1}{\lambda_{m} \mu_{n}} \sum_{\substack{j \in I_{m} \\
(j, k) \in F(\epsilon)}} \sum_{\substack{k \in J_{n} \\
(j, k) \in F(\epsilon)}}\left(g\left(x_{j k}-\xi\right)\right)^{p} \\
\leq\left(\sup g\left(x_{j k}-\xi\right)\right) \frac{1}{\lambda_{m} \mu_{n}}\left(\left|\left\{j \in I_{m}, k \in J_{n}:\left(g\left(x_{j k}-\xi\right)\right)^{p} \geq \epsilon\right\}\right|\right) \\
\leq \frac{M}{\lambda_{m} \mu_{n}}\left|\left\{j \in I_{m}, k \in J_{n}:\left(g\left(x_{j k}-\xi\right)\right)^{p} \geq \epsilon\right\}\right| .
\end{gathered}
$$

We see that the right of above inequality tends to zero as $m, n \rightarrow \infty$, since $\delta_{\lambda, \mu}(F(\epsilon))=0$. Hence, we conclude that $x_{k} \longrightarrow \xi\left[V_{\lambda, \mu}, g\right]_{p}$.

Remark 3.3. If we choose $\lambda_{m}=m$ and $\mu_{n}=n$, then strong $(\lambda, \mu)_{p}$-summablity in a paranormed space is reduced to the notion of strong $p$-Cesàro summablity for double sequences in the same setup, denoted by $\left[C_{1,1}, g\right]_{p}$. Then, we have the following corollary from Theorem 3.3.

Corollary 3.4. Let $(X, g)$ be a paranormed space.

(i) If $p \in(0, \infty)$ and $x_{j k} \longrightarrow \xi\left[C_{1,1}, g\right]_{p}$, then $g\left(S_{2}\right)-\lim x=\xi$.

(ii) If $x=\left(x_{j k}\right) \in \mathcal{L}_{\infty}$ and $g\left(S_{2}\right)$ - $\lim x=\xi$ then $x_{j k} \longrightarrow \xi\left[C_{1,1}, g\right]_{p}(p \in(0, \infty))$.

Theorem 3.5. If a double $x=\left(x_{j k}\right)$ is strongly $(\lambda, \mu)_{p}$-summable or $(\lambda, \mu)$-statistically convergent to $\xi$ in $(X, g)$, then there is a convergent double sequence $y=\left(y_{j k}\right)$ and a $(\lambda, \mu)$-statistically null double sequence $z=\left(z_{j k}\right)$ such that $y=\left(y_{j k}\right)$ is convergent to $\xi$ in Pringsheim's sense, $x=y+z$ and

$$
(P) \lim _{m, n} \frac{1}{\lambda_{m} \mu_{n}}\left|\left\{(j, k), j \in I_{m}, k \in J_{n}: z_{j k} \neq 0\right\}\right|=0 .
$$

Moreover, if a double sequence $x=\left(x_{j k}\right)$ is bounded, then both $\left(y_{j k}\right)$ and $\left(z_{j k}\right)$ are bounded. 
Proof. It is clear from Theorem 3.2 that if a double sequence $x_{j k} \longrightarrow \xi\left[V_{\lambda, \mu}, g\right]_{p}$, then it is $(\lambda, \mu)$-statistically convergent to $\xi$. Let us take $S(0)=0$ and choose a strictly increasing sequence $S(1)<S(2)<S(3)<\ldots$ of positive integers such that

$$
\frac{1}{\lambda_{m} \mu_{n}}\left|\left\{(j, k), j \in I_{m}, k \in J_{n}:\left|x_{j k}-\xi\right| \geq l\right\}\right|<l^{-1},
$$

for $m, n>S(l)$. We are defining $y=\left(y_{j k}\right)$ and $z=\left(z_{j k}\right)$ as follows: Choose $z_{j k}=0$ and $y_{j k}=x_{j k}$ if $S(0)<j, k<S(1)$. Suppose $l \geq 1$ and $S(l)<j, k<S(l+1)$. We now set

$$
y_{j k}=\left\{\begin{array}{l}
x_{j k}, z_{j k}=0 \text { for }\left|x_{j k}-\xi\right|<l^{-1} \\
\xi, z_{j k}=x_{j k}-\xi \text { for }\left|x_{j k}-\xi\right| \geq l^{-1}
\end{array}\right.
$$

Clearly, $x=y+z$ and double sequences $y$ and $z$ are bounded if a double sequence $x$ is bounded. We have to show that $y=\left(y_{j k}\right)$ is convergent to $\xi$ in the Pringsheim's sense. For given $\epsilon>0$, let us choose $l$ such that $\epsilon>1 / l$. We can see that for $j, k>S(l)$, one obtains

$$
\left|y_{j k}-\xi\right|<\epsilon\left(\text { since }\left|y_{j k}-\xi\right|=\left|x_{j k}-\xi\right|<\epsilon\right) \text { if }\left|x_{j k}-\xi\right|<l^{-1}
$$

and

$$
\left|y_{j k}-\xi\right|=|\xi-\xi|=0 \text { if }\left|x_{j k}-\xi\right|>l^{-1} \text {. }
$$

If follows that $\left(y_{j k}\right)$ is convergent to $\xi$ in the Pringsheim's sense. It remains to prove that (3) holds. It is enough to prove that if $\delta>0$ and $l \in \mathbb{N}$ such that $1 / l<\delta$, then

$$
\left|\left\{(j, k), j \in I_{m}, k \in J_{n}: z_{j k} \neq 0\right\}\right|<\delta \forall m, n>S(l) .
$$

As we have seen from the construction that if $S(l)<j, k \leq S(l+1)$ then $z_{j k}=0$ only if $\left|x_{j k}-\xi\right|>1 / l$. It follows that if $S(r)<j, k \leq S(r+1)$, then

$$
\left\{(j, k), j \in I_{m}, k \in J_{n}: z_{j k} \neq 0\right\} \subseteq\left\{(j, k), j \in I_{m}, k \in J_{n}:\left|x_{j k}-\xi\right|>1 / r\right\} .
$$

Consequently, if $S(r)<j, k \leq S(r+1)$ and $r>l$, one obtains

$$
\begin{gathered}
\frac{1}{\lambda_{m} \mu_{n}}\left|\left\{(j, k), j \in I_{m}, k \in J_{n}: z_{j k} \neq 0\right\}\right| \\
\subseteq \frac{1}{\lambda_{m} \mu_{n}}\left|\left\{(j, k), j \in I_{m}, k \in J_{n}:\left|x_{j k}-\xi\right|>1 / r\right\}\right|<1 / r<1 / l<\delta .
\end{gathered}
$$

Thus, we have the following

$$
(P) \lim _{m, n} \frac{1}{\lambda_{m} \mu_{n}}\left|\left\{(j, k), j \in I_{m}, k \in J_{n}: z_{j k} \neq 0\right\}\right|=0 .
$$




\section{REFERENCES}

[1] T. Acar and S. A. Mohiuddine, Statistical $(C, 1)(E, 1)$ summability and Korovkin's theorem, Filomat 30(2) (2016), $387-393$.

[2] A. Alotaibi and A. M. Alroqi, Statistical convergence in a paranormed space, J. Inequal. Appl. 2012 (2012), 39.

[3] A. Alotaibi, M. Mursaleen, S. A. Mohiuddine, Korovkin type approximation theorems for $\sigma$-convergence of double sequences, J. Nonlinear Convex Anal. 16(1) (2015), 183-192.

[4] M. A. Alghamdi and M. Mursaleen, $\lambda$-Statistical convergence in paranormed space, Abstr. Appl. Anal. 2013 (2013), Article ID 264520 .

[5] F. A. Arani, M. E. Gordji and S. Talebi, Statistical convergence of double sequence in paranormed spaces, J. Math. Comput. Sci. 10 (2014), 47-53.

[6] C. Belen and S. A. Mohiuddine, Generalized weighted statistical convergence and application, Appl. Math. Comput. 219 (2013), 9821-9826.

[7] N. L. Braha, H. M. Srivastava and S. A. Mohiuddine, A Korovkin's type approximation theorem for periodic functions via the statistical summability of the generalized de la Vallée Poussin mean, Appl. Math. Comput. 228 (2014), 162-169.

[8] H. Çakalli, On statistical convergence in topological groups, Pure Appl. Math. Sci. 43 (1996), 27-31.

[9] J. S. Connor, The statistical and strong p-Cesàro convergence of sequences, Analysis 8 (1988), 47-63.

[10] O. H. H. Edely, S. A. Mohiuddine and A. K. Noman, Korovkin type approximation theorems obtained through generalized statistical convergence, Appl. Math. Lett. 23 (2010), 1382-1387.

[11] H. Fast, Sur la convergence statistique, Coll. Math. 2 (1951), 241-244.

[12] J. A. Fridy, On statistical convergence, Analysis 5(4) (1985), 301-313.

[13] B. Hazarika and V. Kumar, On asymptotically double lacunary statistical equivalent sequences in ideal context, J. Inequal. Appl. 2013 (2013), 543.

[14] U. Kadak and S. A. Mohiuddine, Generalized statistically almost convergence based on the difference operator which includes the ( $p, q)$-Gamma function and related approximation theorems, Results Math. 73 (2018), 9.

[15] E. Kolk, The statistical convergence in Banach spaces, Tartu Ul. Toime. 928 (1991), 41-52.

[16] I. J. Maddox, Statistical convergence in a locally convex space, Math. Camb. Phil. Soc. 104 (1988), 141-145.

[17] S. A. Mohiuddine, Statistical weighted $A$-summability with application to Korovkin's type approximation theorem, J. Inequal. Appl. 2016 (2016), 101.

[18] S. A. Mohiuddine and B. A. S. Alamri, Generalization of equi-statistical convergence via weighted lacunary sequence with associated Korovkin and Voronovskaya type approximation theorems, Rev. R. Acad. Cienc. Exactas Fs. Nat., Ser. A Mat., RACSAM 113 (3) (2019), 1955-1973.

[19] S. A. Mohiuddine and M. Aiyub, Lacunary statistical convergence in random 2-normed spaces, Appl. Math. Inf. Sci. 6(3) (2012), 581-585.

[20] S. A. Mohiuddine, A. Alotaibi and M. Mursaleen, Statistical convergence of double sequences in locally solid Riesz spaces, Abstr. Appl. Anal. 2012 (2012), 719729.

[21] S. A. Mohiuddine, A. Asiri and B. Hazarika, Weighted statistical convergence through difference operator of sequences of fuzzy numbers with application to fuzzy approximation theorems, Int. J. Gen. Syst. 48(5) (2019), 492-506.

[22] S. A. Mohiuddine and B. Hazarika, Some classes of ideal convergent sequences and generalized difference matrix operator, Filomat 31(6) (2017), 1827-1834

[23] S. A. Mohiuddine, B. Hazarika and A. Alotaibi, On statistical convergence of double sequences of fuzzy valued functions, J. Intell. Fuzzy Syst. 32 (2017), 4331-4342. 
[24] M. Mursaleen, $\lambda$-Statistical convergence, Math. Slovaca 50 (2000), 111-115.

[25] M. Mursaleen, C. Çakan, S. A. Mohiuddine and E. Savaş, Generalized statistical convergence and statistical core of double sequences, Acta Math. Sin. 26(11) (2010), 2131-2144.

[26] M. Mursaleen and O. H. H. Edely, Statistical convergence of double sequences, J. Math. Anal. Appl. 288 (2003), $223-231$.

[27] M. Mursaleen and S. A. Mohiuddine, Statistical convergence of double sequences in intuitionistic fuzzy normed spaces, Chaos Solitons Fractals 41(5) (2009), 2414-2421.

[28] M. Mursaleen and S. A. Mohiuddine, On lacunary statistical convergence with respect to the intuitionistic fuzzy normed space, J. Comput. Appl. Math. 233 (2009), 142-149.

[29] A. Pringsheim, Zur Ttheorie der zweifach unendlichen Zahlenfolgen, Math. Ann. 53 (1900), 289-321.

[30] I. J. Schoenberg, The integrability of certain functions and related summability methods, Amer. Math. Monthly 66 (1959), 361-375.

[31] T. S̆alát, On statistically convergent sequences of real numbers, Math. Slovaca 30 (1980), 139-150.

[32] A. Zygmund, Trigonometrical Series, vol. 5(1935) of Monografýas de Matemáticas, Warszawa-Lwow. 